\title{
EFFICIENT AND NONINTRUSIVE ELECTROPUMPING OF WATER IN NANOTUBES
}

\author{
DAVID OSTLER
}

(Received 9 October 2020; first published online 14 December 2020)

2020 Mathematics subject classification: primary 76M99.

Keywords and phrases: carbon nanotubes, electropumping, functionalisation, molecular dynamics, water.

Electropumping has shown genuine potential as an effective means of inducing a net positive flow of water confined in nanochannels. In this thesis we present the first nonequilibrium molecular dynamics results that demonstrate a net positive flow within a single functionalised carbon nanotube and between concentric carbon nanotubes using electropumping. Electropumping involves the application of a rotating electric field to water in confined geometries to induce a net positive flow. We achieve this by applying a spatially uniform rotating electric field that couples to the water's permanent electric dipole moment. To take advantage of the coupling between the spin angular momentum and the linear momentum we break the symmetry in the single carbon nanotube by asymmetrically functionalising the inner surface of the carbon nanotube with carboxyl groups to induce a net positive flow. For concentric nanotubes, symmetry is broken by functionalising the outer surface of the inner nanotube, again with carboxyl groups. We show that a net flow can be achieved with only a small amount of functionalisation, which can be attributed to a combination of the strongly hydrophilic nature of the carboxyl group combined with the small diameter of the carbon nanotubes. We numerically solve the coupled hydrodynamic momentum equations for concentric nanotubes to show that the nonequilibrium molecular dynamics results are consistent with continuum theory.

We compare the average power dissipation between electropumping, Couette and Poiseuille flows to determine the relative efficiencies of each method of inducing a net positive flow. Adjusting the wall velocity for Couette flow, the rotational electric field amplitude and frequency for electropumping and the external field for Poiseuille flow to ensure that the volumetric flow rate is matched for each nanochannel allows a direct comparison between flow types. We show that while electropumping is four orders of magnitude less efficient than Couette flow, it is more efficient than Poiseuille

Thesis submitted to Swinburne University of Technology in May 2020; degree approved on 3 August 2020; supervisors Billy Todd, Peter Daivis and Federico Frascoli.

(C) 2020 Australian Mathematical Publishing Association Inc. 
flow, suggesting that rather than being a mere novelty, electropumping is actually a far more energetically efficient means of transporting water in nanochannels compared to conventional pressure-driven pumping.

Some of the results in the thesis have been published in [1-3].

\section{References}

[1] D. B. Ostler, S. K. Kannam, P. J. Daivis, F. Frascoli and B. D. Todd, 'Electropumping of water in functionalized carbon nanotubes using rotating electric fields', J. Phys. Chem. C 121 (2017), $28158-28165$.

[2] D. B. Ostler, S. K. Kannam, F. Frascoli, P. J. Daivis and B. D. Todd, 'Inducing a net positive flow in functionalized concentric carbon nanotubes using rotating electric fields', Langmuir 35 (2018), 14742-14749.

[3] D. B. Ostler, S. K. Kannam, F. Frascoli, P. J. Daivis and B. D. Todd, 'Efficiency of electropumping in nanochannels', Nano Lett. 20 (2020), 3396-3402.

DAVID OSTLER, Department of Mathematics,

Swinburne University of Technology, Hawthorn,

Victoria 3122, Australia

e-mail: daveostler@gmail.com 